\title{
Cardiac SPECT/CT Imaging: CT Attenuation Correction and SPECT/CT Hybrid Imaging
}

\section{Yoshimitsu Fukushima* and Shin-ichiro Kumita}

Department of Radiology, Nippon Medical School Hospital, 1-1-5, Sendagi, Bunkyo-ward, Tokyo 113-8603, Japan

\section{Abstract}

Cardiac single photon emission computed tomography (SPECT)/computed tomography (CT) imaging is facilitated by the dissemination of SPECT/CT hybrid systems and dedicated image fusion software. The combined approach of SPECT and CT has various advantages when compared with stand-alone imaging. CT attenuation correction can correct attenuation artifacts in myocardial SPECT and improves diagnostic accuracy for patients with coronary artery disease (CAD). Cardiac SPECT/CT hybrid imaging allows detection of coronary artery stenoses and abnormalities in myocardial perfusion and fatty-acid metabolism in patients with cardiac disease. It has been reported that this modality improves diagnostic accuracy in the detection of CAD compared to the individual imaging techniques. Furthermore, this approach facilitates the detection of hemodynamically significant coronary artery stenoses and the management of revascularization procedures. These abilities of cardiac hybrid SPECT/CT imaging will be further refined validating a range of clinical applications in large-scale clinical trials.

\section{Publication History:}

Received: January 30, 2016

Accepted: July 20, 2016

Published: July 22, 2016

\section{Keywords:}

Cardiac SPECT/CT imaging, CT attenuation correction, Cardiac SPECT/CT hybrid imaging, Myocardial SPECT, Coronary CT angiography, Coronary artery disease

\section{Introduction}

Myocardial single photon emission computed tomography (SPECT) is a well-established diagnostic modality for the evaluation of myocardial ischemia or metabolic disorder in various cardiac diseases as well as for the risk stratification of coronary artery disease (CAD). Specifically, the clinical usefulness of myocardial perfusion imaging, such as stress myocardial perfusion SPECT, for detecting CAD is supported by abundant evidence in literature [1-3]. Recently, the number of non-invasive cardiac imaging tests has steadily increased [4]. This has driven the dissemination of state-of-the-art devices of computed tomography (CT) and nuclear medicine worldwide, as well as facilitated cardiac SPECT/CT hybrid imaging.

However, the diagnostic accuracy of conventional myocardial SPECT without CT has remained suboptimal due to tissue attenuation artifacts caused by the breasts, lateral chest walls, abdomen, and diaphragm $[5,6]$. For myocardial perfusion SPECT, both American Society of Nuclear Cardiology and Society of Nuclear Medicine and Molecular Imaging recommend the incorporation of attenuation correction (AC) to improve diagnostic accuracy [7]. Although stand-alone SPECT scanners require transmission scans by external radionuclide systems to obtain tissue density maps for AC, SPECT/CT hybrid systems can obtain the maps from CT images. The advantages of the computed tomography attenuation correction (CTAC) method include higher quality attenuation maps due to higher photon flux, lower noise, and improved resolution [8]. In addition, CTAC can improve the diagnostic accuracy of myocardial SPECT $[9,10]$.

As for cardiac SPECT/CT hybrid imaging, either SPECT/CT hybrid systems or SPECT/CT image fusion software can be used to create SPECT/CT hybrid images. This imaging has achieved increasing clinical acceptance because it shows the relationship between anatomical information, including the presence of coronary artery lesions, and functional information, such as myocardial perfusion or metabolism [11-14]. The comprehensive findings derived from this imaging modality can provide guidance for appropriate medical or interventional therapies [15]

The present review provides an overview of the most important clinical aspects of cardiac SPECT/CT imaging, specifically on CTAC and cardiac SPECT/CT hybrid imaging. Provided is an outline of relevant literature that discusses upon both issues, including the hardware and software requirements for cardiac SPECT/CT imaging. As research is ongoing and the technique is continuously expanding into other applications, the conceivable clinical applications of cardiac SPECT/CT hybrid imaging will be discovered.

\section{CTAC for Myocardial SPECT}

\section{Attenuation artifacts in myocardial SPECT}

Myocardial SPECT is a widely used and well-established method for the evaluation of myocardial ischemia or metabolic disorder in various cardiac diseases [1-4]. However, the diagnostic accuracy of conventional myocardial SPECT has remained suboptimal due to various artifacts including attenuation artifacts. Non-homogeneous photon attenuation in the thorax is one of the major drawbacks of myocardial SPECT, limiting the diagnostic accuracy, interpretive confidence, and quantification reliability. Consequently, attenuation artifacts may reduce the specificity of myocardial SPECT since regional reduced accumulation due to attenuation artifacts may be misinterpreted as a perfusion or metabolism defect [16]. Figure 1 shows a representative case with an attenuation artifact in the inferoposterior wall. Meanwhile, attenuation artifacts may also reduce the sensitivity of myocardial SPECT because true accumulation defects may be concealed by them [17]. In Figure 2, an attenuation artifact on the myocardial infarction makes diagnosis complicated.

\section{Correction of attenuation artifacts}

Conventionally, attenuation artifacts in myocardial SPECT can be corrected using supine-prone acquisition [18] or attenuation maps obtained using external radionuclide sources [19]. In the past few

${ }^{*}$ Corresponding Author: Dr. Yoshimitsu Fukushima, M.D., Ph.D., Department of Radiology, Nippon Medical School Hospital, 1-1-5, Sendagi, Bunkyo-ward, Tokyo 113-8603, Japan, Tel: +81-3-3822-2131; E-mail: fuku@nms.ac.jp

Citation: Fukushima Y, Kumita S (2016) Cardiac SPECT/CT Imaging: CT Attenuation Correction and SPECT/CT Hybrid Imaging. Int J Radiol Med Imag 2: 113. doi: https://doi.org/10.15344/2456-446X/2016/113

Copyright: ( 2016 Fukushima et al. This is an open-access article distributed under the terms of the Creative Commons Attribution License, which permits unrestricted use, distribution, and reproduction in any medium, provided the original author and source are credited. 
Citation: Fukushima Y, Kumita S (2016) Cardiac SPECT/CT Imaging: CT Attenuation Correction and SPECT/CT Hybrid Imaging. Int J Radiol Med Imag 2: 113. doi: https://doi.org/10.15344/2456-446X/2016/113

Page 2 of 12



Figure 1: Effects of CTAC in healthy participant. In both the conventional NaI and CZT images, the inferoposterior posterolateral, and septal attenuation artifacts are accurately corrected by CTAC.

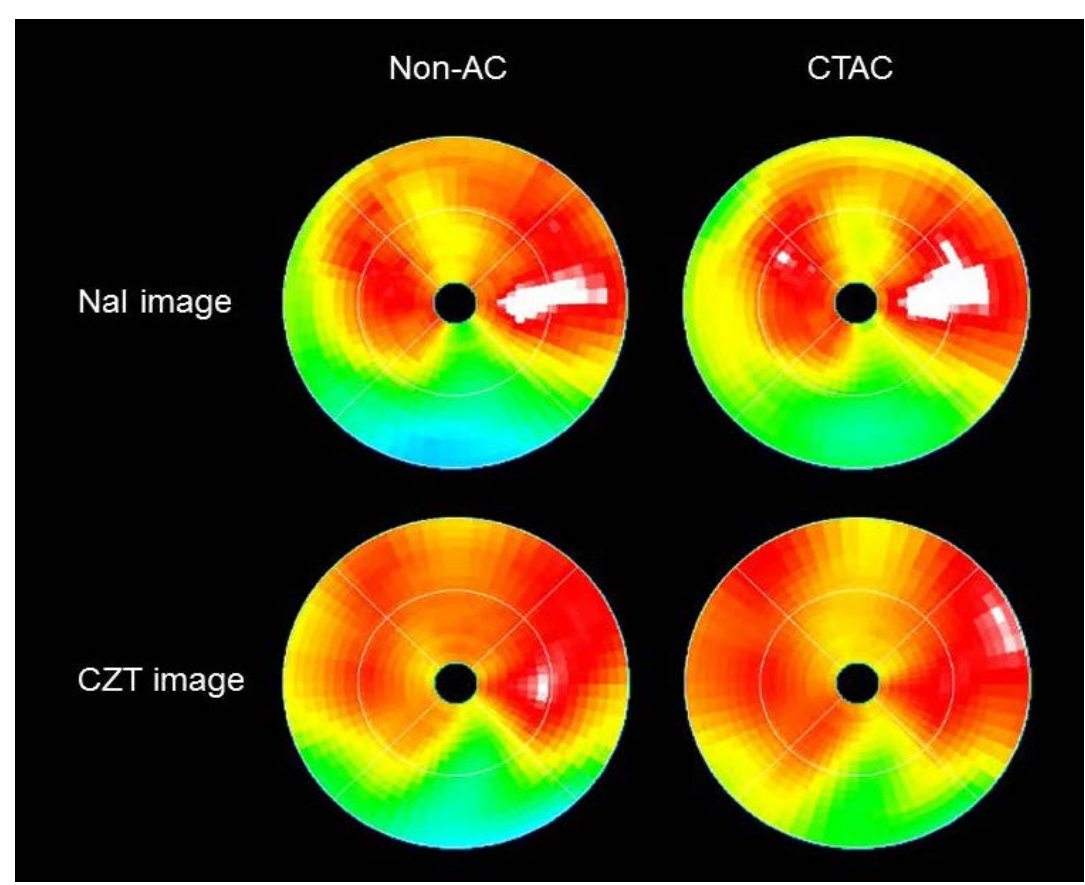

Figure 2: Effects of CTAC in patients with anteroapical myocardial ischemia and inferoposterior myocardial infarction. In both the non-AC images, a myocardial infarction in the inferoposterior wall is overestimated. In the CZT images, an anteroapical myocardial ischemia underestimated in the non-AC image is clearly depicted in the CTAC image. 
decades, however, attenuation maps, derived from unenhanced CT scans, have been introduced as a reliable method for AC of myocardial SPECT for clinical practice [20-22]. Figure 1 shows correction of diaphragmatic attenuation artifacts using CT attenuation maps in myocardial perfusion SPECT images. CTAC is instrumental in distinguishing attenuation artifacts from true myocardial perfusion defects in the inferoposterior wall. However, apical perfusion defect, probably due to anatomical apical thinness or wall motion, is often observed in CTAC images and it should be carefully interpreted. CTAC has been proven to have higher diagnostic accuracy than supine-prone acquisition in myocardial SPECT; these modalities have definitive diagnostic rates of $90 \%$ and $82 \%$, respectively, for significant CAD ( $>50 \%$ coronary artery stenosis in coronary angiograms) [10]. Compared to AC with external radionuclide sources, CTAC has several advantages, such as higher photon flux, no decay of transmission source, shorter scan times, lower noise, and improved resolution $[23,24]$. CTAC can be conducted using a SPECT/CT hybrid system or stand-alone SPECT and CT scanners. Using SPECT/CT hybrid systems increases the specificity of myocardial SPECT to $80-90 \%$ [9]. SPECT/CT studies have shown that low-dose CT acquisitions are also feasible for CTAC [25]. The main limitation of CTAC is misregistration between myocardial SPECT and CT attenuation maps due to respiratory and cardiac motion $[24,26]$. Registration correction using software may be required in cases with $1.3 \pm 0.4$ pixels of misalignment to avoid false accumulation defects [27]. Another limitation of CTAC is potential misalignment between emission and transmission data due to the difference of spatial resolutions, causing the risk of incomplete correction and artificial perfusion defects. Thus, quality control can be used to avoid reconstruction artifacts. PET/CT $[28,29]$ and SPECT/CT $[30,31]$ studies have shown that the frequency of misalignment can be high. Recently, it has been shown that the effects of misalignment are less severe for SPECT/CT than for $\mathrm{PET} / \mathrm{CT}$, mainly because of the low spatial resolution of SPECT [32]. The alignment of emission and transmission data is usually performed manually with a high success rate. Furthermore, automated methods for registration are being developed [33,34].

\section{CTAC for myocardial SPECT using solid-state gamma cameras}

Recently, new gamma cameras, optimized for cardiac imaging and equipped with solid-state detectors, have been introduced for clinical use. Discovery NM 530c or 630 (GE healthcare) and D-SPECT (Spectrum Dynamics), equipped with cadmium-zinc-telluride (CZT) detecting elements, are commercially available [35-38] and a prototype gamma camera: R1-M (Hitachi, Ltd.), equipped with cadmium-telluride detecting elements, is currently being developed $[39,40]$. Compared with conventional NaI gamma cameras, the CZT gamma cameras have multiple advantages, such as improved spatial resolution, high energy resolution, and high sensitivity which reduces patients' discomfort and minimizes motion artifacts $[37,40,41]$. However, since the acquisition using the CZT cameras is performed by positioning gamma-ray detectors on the anterior and left lateral side of the thorax, the myocardial SPECT images show noticeable attenuation artifacts in the septal, inferoposterior, and posterolateral wall of the left ventricular myocardium [42]. The degree of attenuation artifacts in CZT images is markedly higher than in conventional $\mathrm{NaI}$ images (Figure 1). AC using supine-prone acquisition or CTAC is a possible solution $[43,44]$. Although the majority of dedicated CZT gamma cameras do not include an integrated CT scanner, CTAC can be conducted using an external-CT-derived attenuation map and CTAC software. Using CTAC, the diagnostic accuracy of CZT myocardial SPECT can be increased (Figure 1 and 2) [44].

\section{Cardiac SPECT/CT hybrid imaging}

\section{Clinical background}

Development in non-invasive modalities and imaging devices has driven the dissemination of coronary CT angiography (CCTA) and myocardial SPECT for patients with CAD. CCTA is an acceptable alternative to the invasive coronary angiography for the visualization of coronary morphology [45]. However, CCTA solely addresses the morphological findings of coronary arteries and fails to assess the myocardial ischemia caused by coronary artery stenoses [46]. A number of studies have consistently demonstrated that the severity of coronary stenosis is a poor predictor of myocardial ischemia. The same degrees of coronary-artery luminal stenoses may have differing hemodynamic effects among individuals, especially in the moderately stenotic lesions, and collateral vessel formation can alleviate myocardial ischemia $[45,47]$. Similarly, a comparative study of CCTA and myocardial perfusion SPECT has shown that only $32 \%$ of significant coronary stenoses are associated with myocardial ischemia [12]. Furthermore, recent studies using fractional flow reserve have shown that stenoses of 50 to $90 \%$ do not necessarily cause myocardial ischemia $[48,49]$. The prospective nuclear substudy of the COURAGE trial showed that percutaneous coronary intervention (PCI) is effective in patients with myocardial ischemia and that the reduction in myocardial ischemic volume can improve the patients' prognosis. These data indicate that assessing both morphological and functional findings in CAD is crucial in order to offer the optimal treatments to stable CAD patients. Furthermore, cardiac SPECT/CT hybrid imaging can show the direct relationships between the distribution of coronary artery stenoses and myocardial ischemia, improving the diagnostic accuracy.

\section{Role of myocardial SPECT in cardiac hybrid imaging}

In the past few decades, myocardial perfusion SPECT has been established as a reliable non-invasive modality for diagnosing myocardial ischemia, which has sensitivity and specificity of $87-89 \%$ and $73-75 \%$, respectively, for significant CAD ( $>50 \%$ coronary artery stenosis in coronary angiograms) [50]. Furthermore, myocardial perfusion SPECT has an independent prognostic value in various clinical settings, such as stable CAD, risk assessment prior to noncardiac surgery, and acute coronary syndrome (ACS) [51]. Notably, normal myocardial perfusion in patients with stable CAD indicates an excellent, mid-term prognosis; the incidence rate of death or non-fatal myocardial infarction is less than $1 \%$ in a year [1]. However, myocardial SPECT has several limitations. In patients with multivessel CAD or balanced myocardial ischemia, myocardial SPECT may underestimate the severity of myocardial ischemia although the myocardial segment with the severest ischemia is detectable [52-54]. Furthermore, myocardial SPECT cannot detect non-obstructive coronary atherosclerotic lesions, which do not cause myocardial ischemia, but such lesions may cause ACS or develop into obstructive atherosclerotic lesions $[48,55,56]$. In addition to myocardial perfusion imaging, myocardial fatty-acid metabolism can also be observed using ${ }^{123}$ I-beta-methyl-p-iodophenylpentadecanoic acid ( ${ }^{123}$ I-BMIPP). Myocardial fatty-acid metabolism SPECT can visualize myocardial metabolic shift caused by myocardial ischemia and myocardial damage from other causes. This modality is useful for diagnosing relatively-severe myocardial ischemia, such as ACS, and differentiating between ischemic heart disease (IHD) and non-IHD in patients with unknown-cause heart failure [57-60]. 


\section{Role of CCTA in cardiac hybrid imaging}

CCTA has been recognized as a non-invasive imaging modality for coronary morphology as an alternative to the invasive coronary angiography [61-63]. Recent multi-slice CT scanners can obtain high temporal and spatial resolution images with low radiation exposure [64]. Three multi-center studies have demonstrated the high diagnostic accuracy of 64-slice CCTA with a sensitivity and specificity of $85-99 \%$ and $64-90 \%$, respectively. In particular, the high negative predictive value, which is close to $100 \%$, has solidified CCTA as an excellent tool for confirming the absence of CAD in patients with low to intermediate pre-test probability $[62,63]$. Despite its high diagnostic accuracy, CCTA exclusively shows coronary-artery anatomical findings without hemodynamic information. However, CCTA provides coronary arterial wall findings with information about nonobstructive plaques as well as plaque size, composition, and coronary artery remodeling. The additional prognostic value of detecting non-obstructive coronary plaques has been extensively explored. Motoyama et al. reported that low attenuation plaques, which have CT values less than $30 \mathrm{HU}$, with positive vascular remodeling lead to high incidence of future ACS events $[65,66]$. According to Otsuka et al., napkin-ring sign shown on CCTA is strongly associated with future ACS events [67-69]. The non-obstructive coronary lesions with these high-risk plaque characteristics, even those without myocardial ischemia, may indicate certain risks of future ACS events. Thus, CCTA can be a complementary modality of myocardial SPECT for cardiac hybrid imaging.

\section{Cardiac hybrid imaging hardware and software}

As it is widely known, hybrid imaging was started with PET/CT hybrid systems, which were mainly used for the field of oncology. Following this advancement of imaging devices, similar hybrid devices, combining SPECT gamma cameras with multi-detector CT scanners, have been recently introduced. These hybrid devices provide location information equally to both SPECT and CT images and enable highly accurate image fusion of these images [70]. However, the majority of these hybrid devices are available with a multi-detector CT device equipped with 16 or fewer detectors despite the recommended minimum number of CT detectors for CCTA being 64. CT devices with a fewer number of detectors have a low temporal and spatial resolution and cannot provide sufficient image quality. As for cardiac hybrid imaging, the use of hybrid systems is not necessarily required. This is due to the myocardium's larger size not requiring the fine detail of hybrid imaging as well as the difference in respiratory conditions between SPECT and CT acquisitions. Whether a hybrid system is used or not, manual correction of misalignment is crucial to improve the quality of fused images because of the following reasons: cardiac and respiratory motion, size and shape difference of the left ventricle due to the difference in spatial resolution, and the presence or absence of ECG-gating. Therefore, cardiac hybrid imaging can easily be performed using stand-alone systems with fusion software. Fusion software packages are available from several manufacturers that allow fusion of CCTA and SPECT images [11]. The image processing steps for creating fusion images usually consist of image co-registration, extraction of LV epicardium, projection of myocardial tracer accumulation to LV epicardium, extraction of coronary tree, and display of 3D volume rendered SPECT/CTA fusion images. Current software packages allow fusion of these images from different manufacturers. Image fusion processing time takes less than 5 minutes for experienced operators, provided the images are high quality.

\section{Clinical value of cardiac SPECT/CT hybrid imaging}

Traditionally, the diagnosis of CAD is conducted using CCTA or myocardial SPECT individually or side-by-side interpretation using both modalities. However, the distribution of coronary arteries differs considerably among individuals and the standardized myocardial segmentation model for coronary artery territory cannot be applied in up to $72 \%$ of patients [71]. The largest variation in coronary artery distribution is in the dominance of the right coronary artery or left circumflex artery in the inferoposterior and posterolateral wall of the left ventricular myocardium (Figure 3). Therefore, the additional information provided by cardiac SPECT/CT hybrid imaging can be used in combination with myocardial SPECT and CCTA to ascertain the interpretation of coronary artery territory in different individuals. Furthermore, cardiac SPECT/CT hybrid imaging can show the direct relationships between the distribution of coronary artery stenoses and myocardial ischemia.

The feasibility and clinical demand of non-invasive cardiac SPECT/ CT hybrid imaging, processed using a self-built algorithm, were initially realized by Nakajo et al. [72]. Rispler et al. reported drastic improvement in specificity (from 63 to $95 \%$ ) and positive predictive value (PPV) (from 31 to 77\%) with SPECT/CCTA compared to CCTA alone [73]. Sato et al. showed that supplemental SPECT information, in non-evaluable coronary artery territory on CCTA, significantly improved specificity and PPV (from 80 to $92 \%$ and from 69 to $85 \%$, respectively) [74]. However, these studies have several limitations, including the limited number of patients, the variety of hybrid systems, and the lack of a unified reference standard.

Three studies have specifically mentioned the additional value of cardiac hybrid imaging in comparisons of myocardial perfusion SPECT and CCTA side-by-side analysis with cardiac SPECT/CT fusion imaging. These reported that the use of cardiac SPECT/CT hybrid imaging decreased the indeterminate diagnostic rate and consequently increased the conclusive diagnostic rate compared to side-by-side interpretations in patients with CAD. These patients, who are often found to have multivessel disease (MVD), moderate coronary stenoses, or branch lesions, also often see the greatest diagnostic improvement in regard to these findings via cardiac SPECT/CT fusion imaging (Figure 4 and 5) [75-77]. Hybrid cardiac imaging can facilitate the detection of hemodynamically significant coronary artery stenosis, leading to appropriate choices of therapeutic methods. Furthermore, confirming hemodynamically non-significant stenosis can avoid unnecessary stent implantations, which may dissipate limited medical resources.

Several studies have revealed the prognostic performance of cardiac SPECT/CT hybrid imaging for patients with CAD. van Werkhoven et al. has reported that significant coronary artery stenoses and noncalcified plaques on CCTA provided additional prognostic value over myocardial perfusion SPECT alone [78]. Furthermore, prognostic information of cardiac SPECT/CT hybrid imaging has been reported by Pazhenkottil, et al. [79]. In this study, 324 consecutive patients were divided into three groups: stenoses by CCTA and matched reversible SPECT defects, unmatched CCTA and SPECT findings, and no findings by CCTA or SPECT. Corresponding matched hybrid image findings were associated with a significantly higher event rate (death or myocardial infarction) $(\mathrm{p}<0.005)$ and proved to be an independent predictor for major adverse cardiac events. The annual event rates were $6.0,2.8$, and $1.3 \%$ for patients with matched, unmatched, and no findings, respectively. 
Citation: Fukushima Y, Kumita S (2016) Cardiac SPECT/CT Imaging: CT Attenuation Correction and SPECT/CT Hybrid Imaging. Int J Radiol Med Imag 2: 113. doi: https://doi.org/10.15344/2456-446X/2016/113

Page 5 of 12

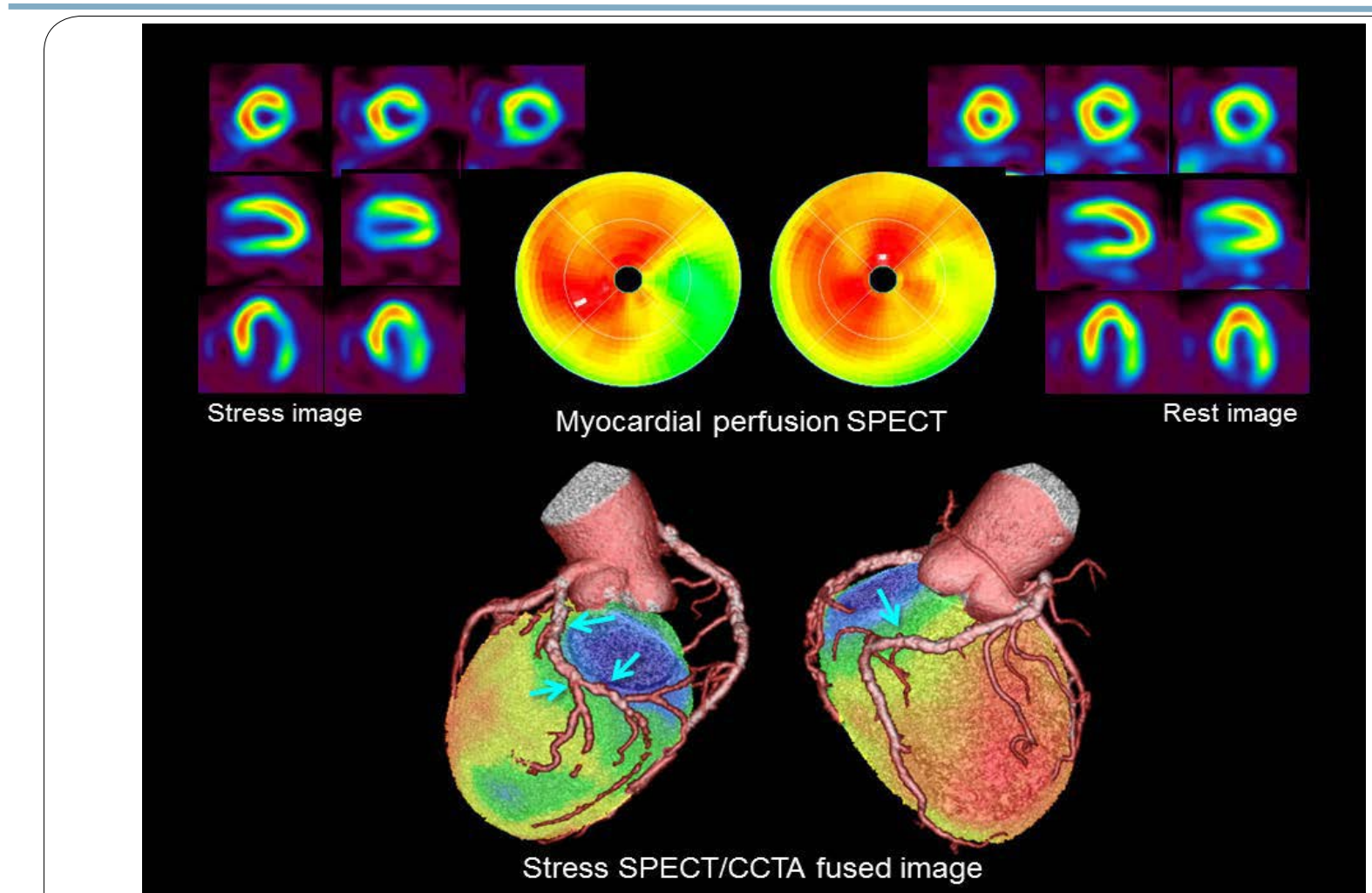

Figure 3: Case of 53-year-old man with LCX dominant and multivessel disease. CCTA revealed a $>75 \%$ stenosis in the mid RCA, a $>50 \%$ stenosis in the proximal LCX, a $>75 \%$ stenosis in the obtuse marginal branch, and a $>75 \%$ stenosis inthe mid LCX. Exercise-stress myocardial perfusion SPECT using ${ }^{99 \mathrm{~m} T c-t e t r o f o s m i n ~(n o n-A C ~ i m a g e s) ~ s h o w e d ~ p a r t i a l l y ~ r e v e r s i b l e ~ p e r f u s i o n ~}$ defects in the lateral and inferoposterior wall. On hybrid SPECT/CT, the lateral and inferoposterior ischemia was located in the LCX territory, while the RCA territory did not show any perfusion defects.

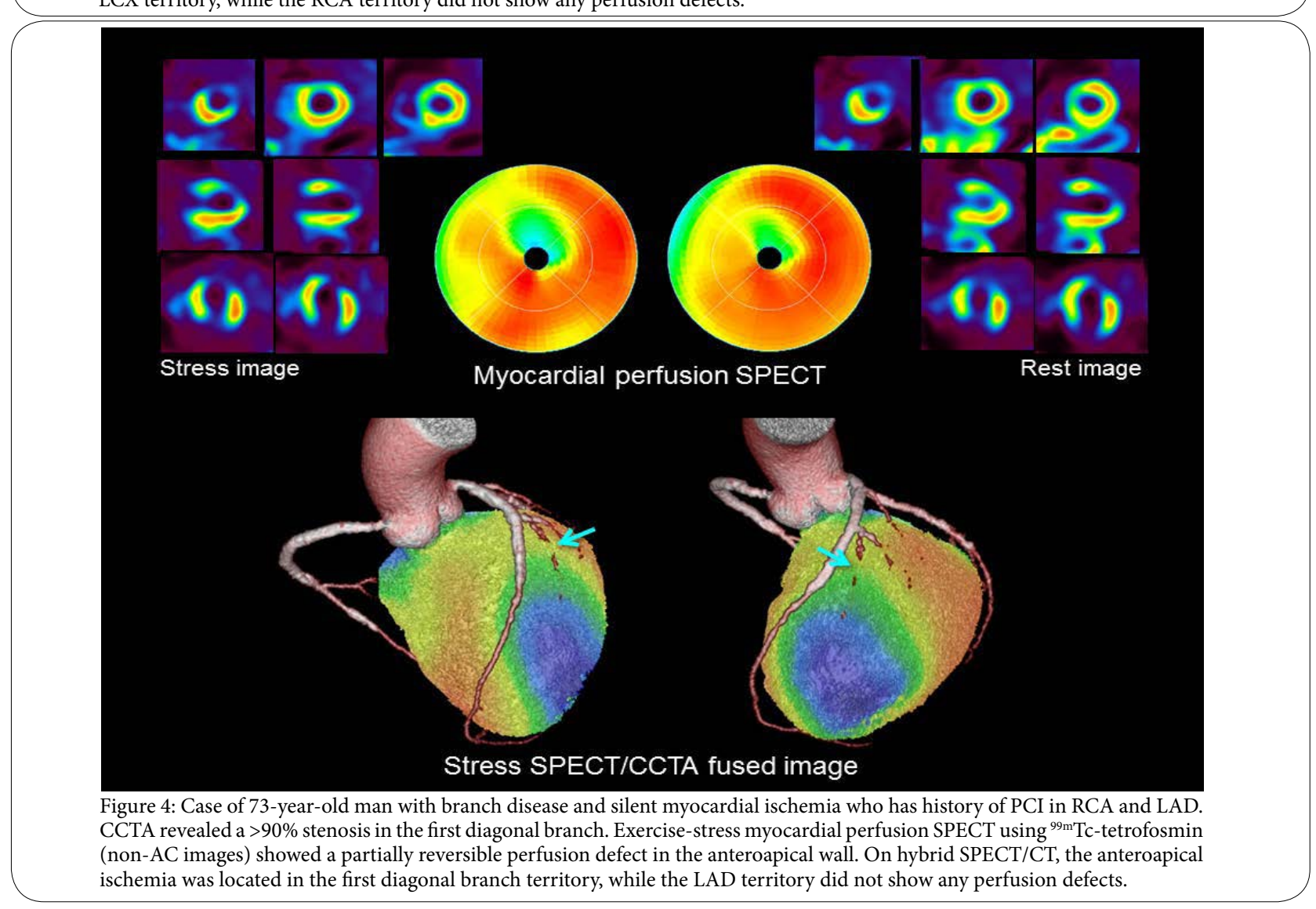




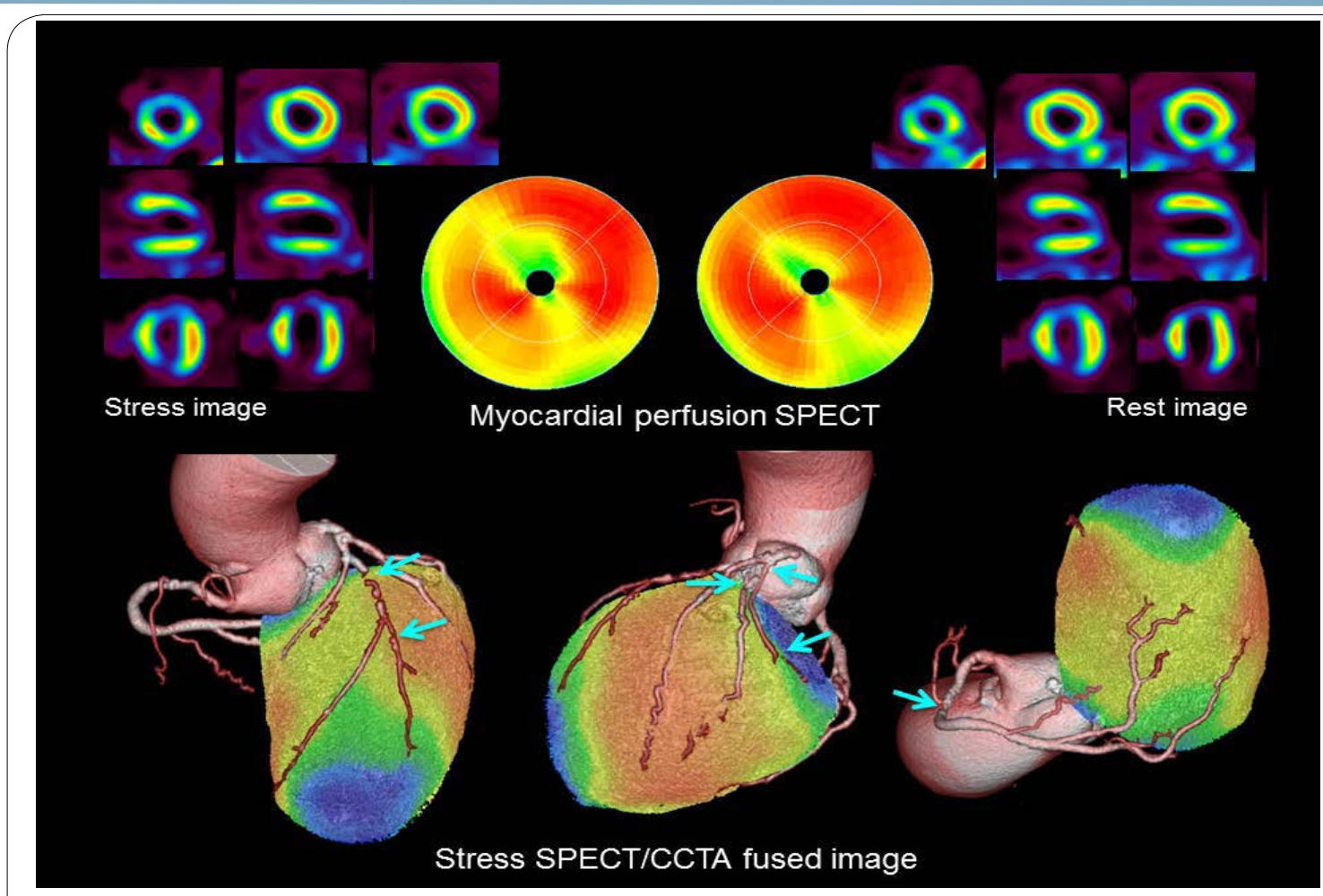

Figure 5: Case of 78-year-old woman with multivessel disease and chronic total occlusion. CCTA revealed a $>75 \%$ stenosis in the mid RCA, a chronic total occlusion in the proximal-mid LAD, a $>90 \%$ stenosis in the first diagonal branch, a $>75 \%$ stenosis in the proximal LCX, a $>75 \%$ stenosis in the obtuse marginal branch, and a $>75 \%$ stenosis in the mid LCX. Adenosine-stress myocardial perfusion SPECT using ${ }^{99 \mathrm{~m}}$ Tc-tetrofosmin (non-AC images) showed a partially reversible perfusion defect in the anteroapical wall and a fixed defect in the inferoposterior wall. On hybrid SPECT/CT, the anteroapical myocardial ischemia was located in the LAD and first diagonal branch territory and the inferoposterior myocardial infarction was situated in the RCA territory.

Two studies have shown that cardiac hybrid imaging has a strong impact on the choice of therapeutic methods for CAD. Follow-up was confined to the first 60 days after hybrid imaging, as this allows for the best assessment treatment strategy decisions. Pazhenkottil et al. reported that revascularization rates within 60 days were 41, 11, and $0 \%$ for matched, unmatched, and no findings, respectively $(\mathrm{p}<$ 0.001 ) [80]. In a similar study by Schaap et al., revascularization rates were even higher, with 90,31 and $0 \%$ for matched, unmatched, and no findings, respectively $(p<0.001)[81]$. These results were expanded upon in the SPARC study, which showed $38-61 \%$ of patients with the most severe findings on CCTA or myocardial perfusion SPECT did not need invasive coronary angiography [82]. Therefore, non-invasive cardiac hybrid imaging may facilitate optimal patient management for patients with CAD.

\section{Coronary artery calcium scoring for hybrid imaging}

Presence of coronary artery calcification (CAC) is a strong indicator of CAD as well as fatal and non-fatal cardiovascular events $[83,84]$. CAC is considered an active, regulated process caused by coronary risk factors and consequent plaque inflammation [85]. The amount of coronary artery calcium, which is represented by coronary artery calcium score (CACS), is directly related to the extent of coronary atherosclerotic plaque burden [86]. CAC is usually quantified on dedicated $3 \mathrm{~mm}$ sliced, plain, and ECG-gated CT scans [87]. CACS might be more accessible with SPECT/CT hybrid systems, as CT scans with these systems are usually performed with low-radiation exposure and without contrast media. Several studies have shown the increasing prognostic value of CACS above traditional cardiovascular risk factors, suggesting that CACS is a useful clinical marker to predict cardiovascular risk in patients with CAD [88]. Using hybrid imaging, the clinical value of integrating CACS and myocardial perfusion SPECT has been realized.

Schepis et al. presented a comparative investigation between myocardial perfusion SPECT, CACS, and invasive coronary angiography in 77 symptomatic patients with intermediate CAD risk [89]. CACS was closely related to the presence of angiographic CAD and severity of myocardial ischemia. Furthermore, the supplemental use of CACS (the optimal cut-off value of CACS was 709) with myocardial perfusion SPECT increased the sensitivity from 76 to $86 \%$ and NPV from 76 to $83 \%$ compared to myocardial perfusion SPECT alone. The supplemental use of CACS may help detect multivessel disease in patient's with pseudonormal myocardial perfusion.

Ghadri et al. presented the adjunct, prognostic value of CACS over myocardial perfusion SPECT using SPECT-CAC hybrid imaging in the REPROSPECT study [90]. Between three groups (matched positive SPECT and CACS findings, unmatched SPECT and CACS findings, and matched negative SPECT and CACS findings), the matched positive group had the most unfavorable outcome ( $p<$ 0.001 for MACE and $\mathrm{p}<0.01$ for death and myocardial infarction).

\section{Radiation exposure}

SPECT and CT are imaging modalities, which entail ionizing radiation exposure. Radiation-induced stochastic effects, such as carcinogenic risk, is directly proportional to radiation dose based on the linear no-threshold model, and unnecessary radiation exposure in 
patients who undergo medical image procedures should be avoided. Radiation exposure dose remarkably varies depending on imaging modalities, radionuclides, and imaging protocols. A study by Hausleiter et al. reported effective radiation doses in early CCTA with 64-slice CT of up to $21.4 \mathrm{mSv}$ [91]. However, patients' radiation exposure from the examination has reduced considerably by $30-90 \%$ because of new image acquisition protocols, in particular the ECG-gated tube current modulation, body-mass-index-adapted tube voltage modulation, and prospective ECG-triggered sequential scanning $[92,93]$. The most recent scanning protocols, using iterative reconstruction, have achieved the reduction of dosages to $0.78-0.99$ $\mathrm{mSv}$ [94].

Similarly, myocardial perfusion SPECT using ${ }^{201} \mathrm{Tl}$ may reach effective radiation doses up to $20-30 \mathrm{mSv}$ [91]. Shorter-half-life perfusion tracers based on ${ }^{99 \mathrm{~m}} \mathrm{Tc}$ allow for a much lower radiation exposure of approximately $9.3 \mathrm{mSv}$ for one-day rest and stress tests [91]. The current introduction of solid-state detectors based on CZT may allow for further reduction of radiation exposure due to a higher detector sensitivity and improved energy resolution [95].

Recently, there have been growing concerns about radiation exposure in medical imaging tests due to the increase of CT scans in the clinical setting [4]. The reduction of radiation exposure must be balanced with maintaining a high level of image quality and diagnostic performance. Particularly, in the field of cardiac non-invasive imaging, drastic reductions of radiation exposure have been achieved through optimized image acquisition protocols and advanced imaging devices, thus facilitating the use of hybrid imaging in the clinical setting [92]. A recent report has documented the feasibility of a stress-only SPECT/CCTA hybrid imaging test with a cumulative radiation dose of $5.4 \mathrm{mSv}$ [96]. Recently, tests to determine maximal dose reduction without compromising image quality were carried out and concluded that ultra-low dose $(<190 \mathrm{MBq})$ myocardial perfusion imaging with short imaging times $(<6 \mathrm{~min})$ is feasible using a hybrid CZT SPECT/ CT camera without compromising image quality [97].

\section{Clinical indications for hybrid imaging}

For diagnosing CAD, either CCTA or myocardial perfusion SPECT is usually chosen for the initial imaging test. As an initial imaging test, CCTA can be chosen for patients with low to intermediate pretest probability and myocardial perfusion SPECT can be chosen for patients with a slightly higher pre-test probability. The presence or absence of CAD can be confirmed with a single non-invasive modality in a large segment of patients and a second non-invasive test may be performed if the first test was inconclusive or equivocal. In addition, myocardial perfusion SPECT is commonly performed on high pre-test probability patients to locate ischemia. However, radiation exposure and examination costs will each increase with the use of both morphological and functional imaging. Therefore, hybrid imaging testing should be carefully considered, and used only if additional value of the imaging is highly expected.

\section{Intermediate pre-test likelihood}

Patients with intermediate pretest likelihood are likely to have inconclusive results in the first non-invasive imaging; these results are usually moderate stenoses in CCTA and equivocal accumulation defects in myocardial SPECT [98]. In this situation, additional imaging may be instrumental to confirm the diagnosis. In patients with moderate coronary stenoses in CCTA, additional myocardial
SPECT can prove whether the stenotic lesion is hemodynamically significant or not. In patients with equivocal accumulation defects in myocardial SPECT, additional CCTA can facilitate a diagnosis, which is usually CAD, non-ischemic heart disease, or artifacts. In both cases, sequential hybrid imaging can be of assistance to formulate therapeutic strategies.

\section{Side branch lesions}

While side branches are often regarded as prognostically insignificant, they often have variations in their size and distribution, which can aid in prognosis. In the case of side branches being unusually large, side branch lesions can cause significant myocardial ischemia. The myocardial ischemia caused by side branch lesions may be difficult to distinguish from that caused by main branches: especially, myocardial ischemia caused by a diagonal branch stenosis, which might be mistaken as that caused by a left anterior descending artery stenosis (Figure 4). Cardiac SPECT/CT hybrid imaging provides accurate coregistration of coronary arteries and perfusion defects and hence allows detection of hemodynamically significant side branch lesions which are targets of revascularization [75].

\section{Multivessel disease}

Patients with MVD have an increased risk for future cardiac events and thus are more likely to undergo revascularization to improve their prognosis. CCTA has revealed that patients with MVD account for a significant portion of those with CAD. Since the majority of patients with three-vessel disease often have less flow-limiting lesions, cardiac hybrid imaging may be advantageous to detect hemodynamically significant coronary artery stenoses (Figure 5) $[76,99]$. As described above, cardiac hybrid imaging may allow for selective revascularization for hemodynamically significant lesions in patients with MVD, avoiding overtreatments with revascularization that may cause adverse sequelae.

\section{Chronic total occlusions}

The implementation of PCI for patients with chronic total occlusions (CTO) is usually associated with a high probability of complications and an increase in the dose of contrast media and radiation exposure. Therefore, myocardial ischemia in the territory of occluded coronary arteries should be proven before PCI. Cardiac hybrid imaging can visualize the morphological information of the lesions, such as the length of the lesion, degree of calcification, and collateral formation, and accurately detect myocardial ischemia in the territory of occluded coronary arteries. Comprehension of the lesion condition prior to PCI might be helpful to improve the procedural success rate [100].

\section{Post coronary artery bypass grafting}

Coronary artery bypass grafting (CABG) is an effective revascularization procedure for patients with advanced CAD. However, graft occlusion or the development of stenoses in the native coronary artery and consequent myocardial ischemia can occur in some cases after the operation (Figure 6) [101,102]. In patients who underwent CABG, multiple coronary stenoses often exist, and the hemodynamic status of the entire coronary artery might be complicated. Since the number of patients with a history of CABG and an indication for revascularization procedures, such as PCI or additional CABG, has been steadily increasing, cardiac SPECT/CT hybrid imaging may play an important role in determining therapeutic strategies [103]. 
Citation: Fukushima Y, Kumita S (2016) Cardiac SPECT/CT Imaging: CT Attenuation Correction and SPECT/CT Hybrid Imaging. Int J Radiol Med Imag 2: 113. doi: https://doi.org/10.15344/2456-446X/2016/113

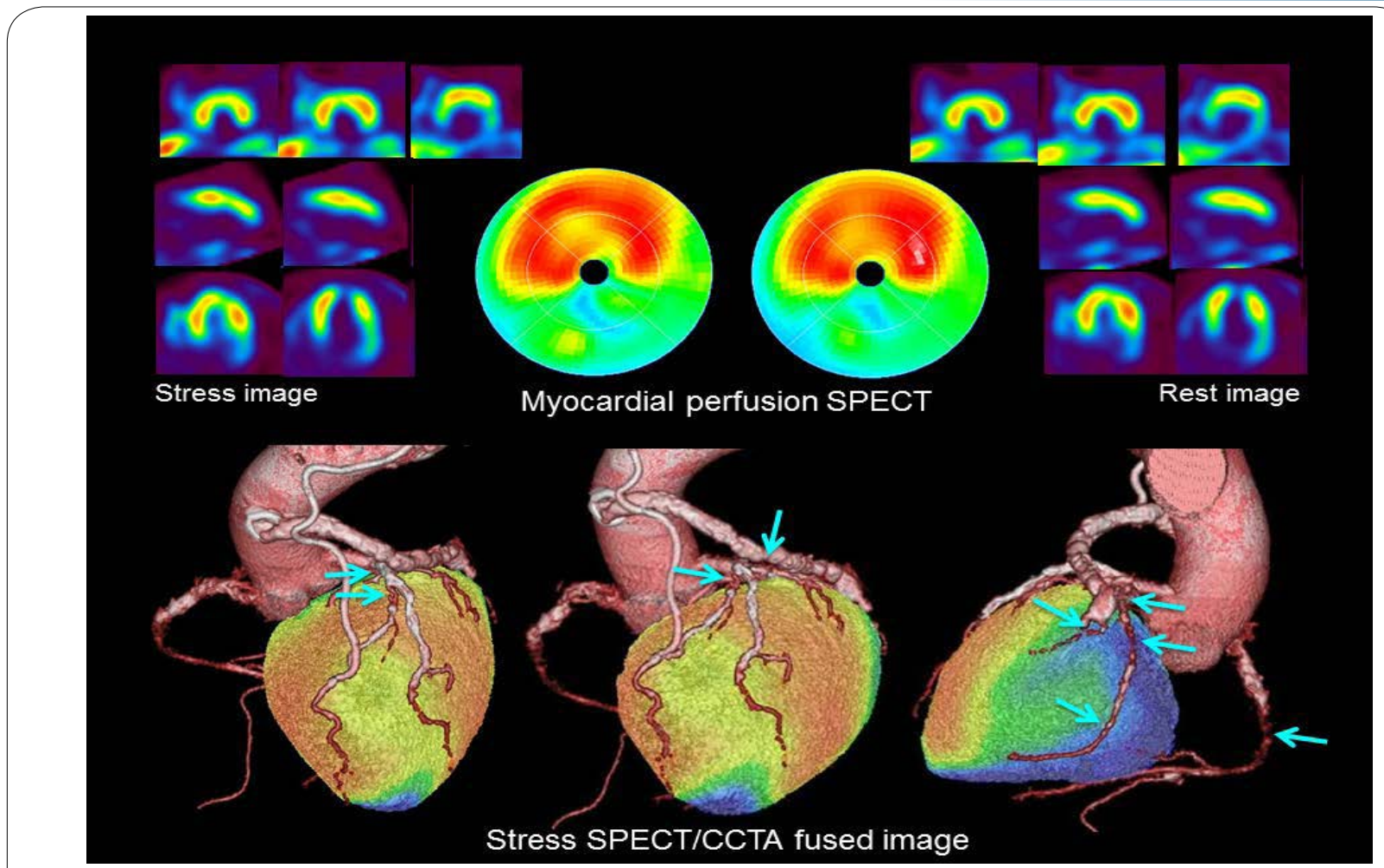

Figure 6: Case of 70-year-old man with effort angina pectoris who has history of myocardial infarction and CABG (LITA-LAD, AoSVG-PL, and GEA-\#4PD). Coronary angiography showed a CTO in the mid RCA, a 99\% stenosis in the proximal-mid LAD, a 90\% stenosis in the proximal LCX, a CTO in the mid LCX, and a 90\% stenosis in the PL. All the bypass grafts were patent. Exercise-stress myocardial perfusion SPECT using ${ }^{99 \mathrm{~m} T c-t e t r o f o s m i n ~(n o n-A C ~ i m a g e s) ~ s h o w e d ~ m y o c a r d i a l ~ i s c h e m i a ~ o n l y ~ i n ~ t h e ~ a n t e r i o r ~ w a l l . ~ O n ~}$ hybrid SPECT/CT, the anterior myocardial ischemia was located in both the first and second diagonal branch territories and the stenotic lesion in the proximal LAD was confirmed to be a culprit lesion.

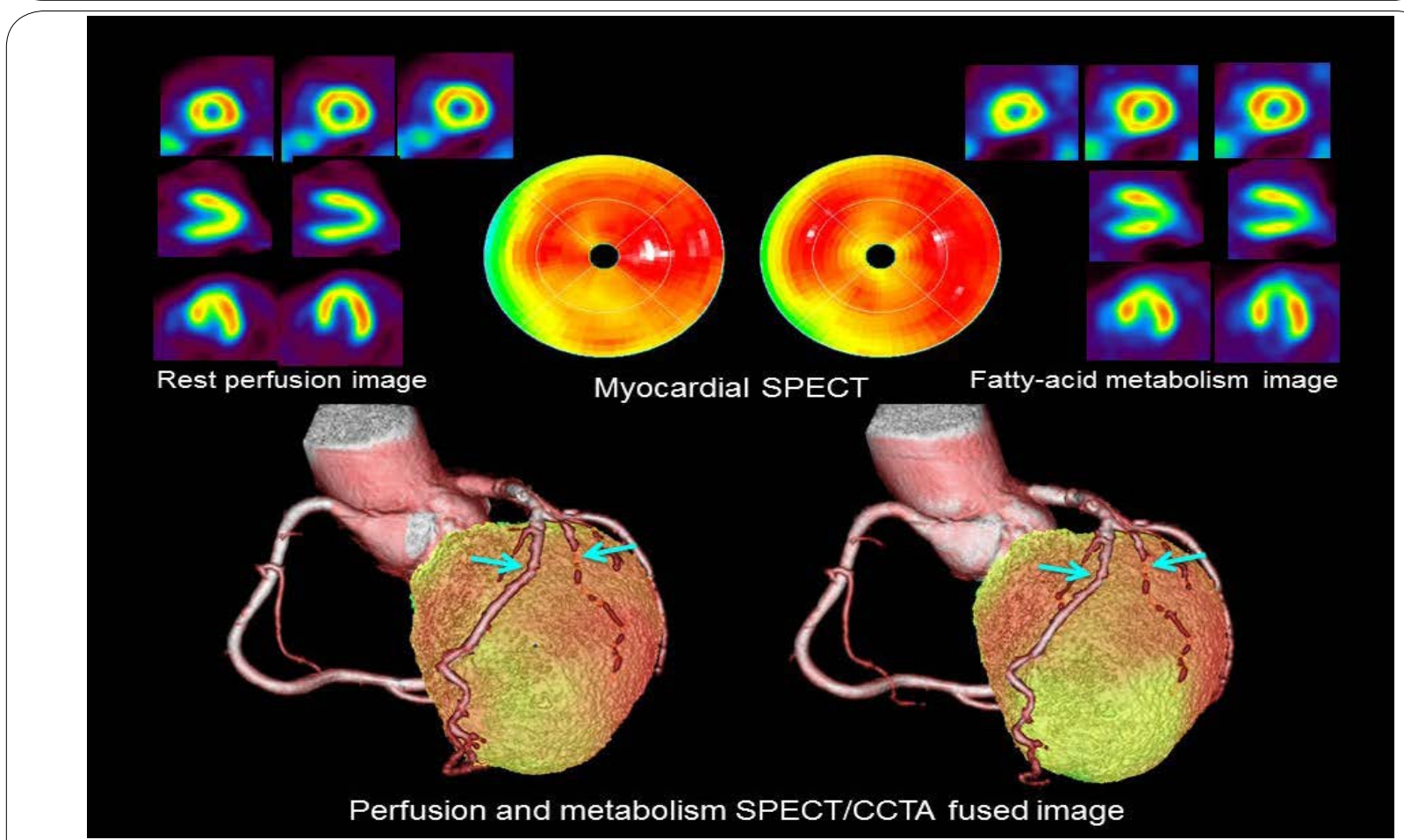

Figure 7: Case of 73-year-old man with unknown-cause acute heart failure. Since coronary angiography revealed a $>75 \%$ stenosis in the mid LAD and a CTO in the first diagonal branch, ischemic heart failure was initially suspected. However, on hybrid SPECT/CT using rest perfusion images with ${ }^{99 \mathrm{~m}} \mathrm{Tc}$-sestamibi and fatty-acid metabolism images with ${ }^{123} \mathrm{I}$-BMIPP (non-AC images), anteroapical myocardial damage was not related to either the LAD nor first diagonal branch territory. The diagnosis was eventually confirmed as takotsubo cardiomyopathy. 


\section{Differential diagnosis in patients with unknown-cause heart failure}

In patients with unknown-cause heart failure, ascertaining the cause is crucial to determine the therapeutic strategy. Myocardial perfusion SPECT can confirm myocardial ischemia and myocardial fatty-acid metabolism SPECT can reveal myocardial damage of any various cause, such as takotsubo cardiomyopathy, cardiac sarcoidosis, and hypertrophic cardiomyopathy $[60,104]$. In the case of multiple accumulation defects in myocardial SPECT images, differentiating between IHD and non-IHD is often difficult. However, cardiac SPECT/CCTA fused images can show the distribution of coronary arteries and accumulation defects, which can be instrumental to distinguish the diagnoses of IHD and non-IHD (Figure 7) [105].

\section{Conclusion}

Cardiac SPECT/CT hybrid imaging is facilitated by the dissemination of state-of-the-art devices of nuclear medicine and CT, as well as by SPECT/CT hybrid systems and dedicated software. The combined approach of SPECT and CT has various advantages when compared with stand-alone imaging. CTAC can correct attenuation artifacts in myocardial SPECT and can improve its diagnostic accuracy. As for cardiac SPECT/CT hybrid imaging, a comprehensive approach, with morphological and functional assessment, may enhance diagnostic ability, improve risk stratification, and guide management strategies for patients with CAD. These abilities of cardiac SPECT/CT imaging will be further refined validating a range of clinical applications in large-scale clinical trials.

\section{Conflicts of Interest}

The author(s) have no conflicts of interest to declare.

\section{Author Contributions}

The author substantially contributed to the literature review, drafting the manuscript and approve the final version of the manuscript.

\section{References}

1. Hachamovitch R, Hayes SW, Friedman JD, Cohen I, Berman DS (2003) Comparison of the short-term survival benefit associated with revascularization compared with medical therapy in patients with no prior coronary artery disease undergoing stress myocardial perfusion single photon emission computed tomography. Circulation 07: 2900-2907.

2. Hendel RC, Berman DS, Di Carli MF, Heidenreich PA, Henkin RE, et al (2009) ACCF/ASNC/ACR/AHA/ASE/SCCT/SCMR/SNM 2009 Appropriate Use Criteria for Cardiac Radionuclide Imaging: A Report of the American College of Cardiology Foundation Appropriate Use Criteria Task Force, the American Society of Nuclear Cardiology, the American College of Radiology, the American Heart Association, the American Society of Echocardiography, the Society of Cardiovascular Computed Tomography, the Society for Cardiovascular Magnetic Resonance, and the Society of Nuclear Medicine. J Am Coll Cardiol 53: 2201-2229.

3. Tamaki N, Morita K, Kuge Y, Tsukamoto E (2000) The role of fatty acids in cardiac imaging. J Nucl Med 41: 1525-1534.

4. Fazel R, Krumholz HM, Wang Y, Ross JS, Chen J, et al. (2009) Exposure to low-dose ionizing radiation from medical imaging procedures. $\mathrm{N}$ Engl $\mathrm{J}$ Med 361: 849-857

5. DePuey EG (1994) How to detect and avoid myocardial perfusion SPECT artifacts. J Nucl Med 35: 699-702.

6. Desmarais RL, Kaul S, Watson DD, Beller GA (1993) Do false positive thallium-201 scans lead to unnecessary catheterization? Outcome of patients with perfusion defects on quantitative planar thallium- 201 scintigraphy. J Am Coll Cardiol 21:1058-1063.
7. Heller GV, Links J, Bateman TM, Ziffer JA, Ficaro E, et al. (2004) American Society of Nuclear Cardiology and Society of Nuclear Medicine joint position statement: attenuation correction of myocardial perfusion SPECT scintigraphy. J Nucl Cardio 11: 229-230.

8. Tonge CM, Manoharan M, Lawson RS, Shields RA, Prescott MC (2005) Attenuation correction of myocardial SPECT studies using low resolution computed tomography images. Nucl Med Commun 26: 231-237.

9. Masood Y, Liu YH, Depuey G, Taillefer R, Araujo LI, et al. (2005) Clinical validation of SPECT attenuation correction using $\mathrm{x}$-ray computed tomography-derived attenuation maps: multicenter clinical trial with angiographic correlation. J Nucl Cardiol 12: 676-686.

10. Malkerneker D, Brenner R, Martin WH, Sampson UK, Feurer ID, et al (2007) CT-based attenuation correction versus prone imaging to decrease equivocal interpretations of rest/stress Tc-99m tetrofosmin SPECT MPI. J Nucl Cardiol 14: 314-323.

11. Gaemperli O, Schepis T, Kalff V, Namdar M, Valenta I, et al. (2007) Validation of a new cardiac image fusion software for three-dimensional integration of myocardial perfusion SPECT and stand-alone 64-slice CT angiography. Eur J Nucl Med Mol Imaging 34: 1097-1106.

12. Gaemperli O, Schepis T, Valenta I, Koepfli P, Husmann L, et al. (2008) Functionally relevant coronary artery disease: comparison of 64-section CT angiography with myocardial perfusion SPECT. Radiology 248: 414-423.

13. Matsuo S, Nakajima K, Akhter N, Wakabayashi H, Taki J, et al. (2009) Clinical usefulness of novel cardiac MDCT/SPECT fusion image. Ann Nucl Med 23: 579-586.

14. Kiriyama T, Toba M, Fukushima $\mathrm{Y}$, Hayashi $\mathrm{H}$, Takano $\mathrm{H}$, et al. (2011) Discordance between the morphological and physiological information of 64-slice MSCT coronary angiography and myocardial perfusion imaging in patients with intermediate to high probability of coronary artery disease. Circ J 75: 1670-1677.

15. Koukouraki S, Pagonidis K, Perisinakis K, Klinaki I, Stathaki M, et al. (2013) Hybrid cardiac imaging: insights in the dilemma of the appropriate clinical management of patients with suspected coronary artery disease. Eur $\mathrm{J}$ Radiol 82: 281-287.

16. DePuey EG, Garcia EV (1989) Optimal specificity of thallium-201 SPECT through recognition of imaging artifacts. J Nucl Med 30: 441-449.

17. Beller GA (1991) Diagnostic accuracy of thallium-201 myocardial perfusion imaging. Circulation 84: 11-6.

18. Nishina H, Slomka PJ, Abidov A, Yoda S, Akincioglu C, et al. (2006) Combined supine and prone quantitative myocardial perfusion SPECT: method development and clinical validation in patients with no known coronary artery disease. J Nucl Med 47: 51-58.

19. Tan P, Bailey DL, Meikle SR, Eberl S, Fulton RR, et al. (1993) A scanning line source for simultaneous emission and transmission measurements in SPECT. J Nucl Med 34: 1752-1760.

20. Fricke E, Fricke $H$, Weise R, Kammeier A, Hagedorn R, et al. (2005) Attenuation correction of myocardial SPECT perfusion images with lowdose CT: evaluation of the method by comparison with perfusion PET. J Nucl Med 46: 736-744.

21. Bocher M, Balan A, Krausz Y, Shrem Y, Lonn A, Wilk M, et al. (2000) Gamma camera-mounted anatomical X-ray tomography: technology, system characteristics and first images. Eur J Nucl Med 27: 619-627.

22. Lang TF, Hasegawa BH, Liew SC, Brown JK, Blankespoor SC, et al. (1992) Description of a prototype emission-transmission computed tomography imaging system. J Nucl Med 33: 1881-1887.

23. Koepfli P, Hany TF, Wyss CA, Namdar M, Burger C, et al. (2004) CT attenuation correction for myocardial perfusion quantification using a PET/ CT hybrid scanner. J Nucl Med 45: 537-542.

24. O'Connor MK, Kemp BJ (2006) Single-photon emission computed tomography/computed tomography: basic instrumentation and innovations. Semin Nucl Med 36: 258-266.

25. Preuss R, Weise R, Lindner O, Fricke E, Fricke H, et al. (2008) Optimisation of protocol for low dose CT-derived attenuation correction in myocardia perfusion SPECT imaging. Eur J Nucl Med Mol Imaging 35: 1133-1141.

26. Madsen MT (2007) Recent advances in SPECT imaging. J Nucl Med 48: 661-673. 
27. Fricke H, Fricke E, Weise R, Kammeier A, Lindner O, et al. (2004) A method to remove artifacts in attenuation-corrected myocardial perfusion SPECT Introduced by misalignment between emission scan and CT-derived attenuation maps. J Nucl Med 45: 1619-1625.

28. Martinez-Möller A, Souvatzoglou M, Navab N, Schwaiger M, Nekolla SG (2007) Artifacts from misaligned CT in cardiac perfusion PET/CT studies: frequency, effects, and potential solutions. J Nucl Med 48: 188-193.

29. Gould KL, Pan T, Loghin C, Johnson NP, Guha A, et al. (2007) Frequent diagnostic errors in cardiac PET/CT due to misregistration of CT attenuation and emission PET images: a definitive analysis of causes, consequences, and corrections. J Nucl Med 48: 1112-1121.

30. Goetze S, Brown TL, Lavely WC, Zhang Z, Bengel FM (2007) Attenuation correction in myocardial perfusion SPECT/CT: effects of misregistration and value of reregistration. $\mathrm{J} \mathrm{Nucl}$ Med 48: 1090-1095.

31. Kennedy JA, Israel O, Frenkel A (2009) Directions and magnitudes of misregistration of CT attenuation-corrected myocardial perfusion studies: incidence, impact on image quality, and guidance for reregistration. J Nucl Med 50: 1471-1478.

32. McQuaid SJ, Hutton BF (2008) Sources of attenuation-correction artefacts in cardiac PET/CT and SPECT/CT. Eur J Nucl Med Mol Imaging 35: 11171123.

33. Chen J, Caputlu-Wilson SF, Shi H, Galt JR, Faber TL, et al. (2006) Automated quality control of emission-transmission misalignment for attenuation correction in myocardial perfusion imaging with SPECT-CT systems. J Nucl Cardiol 13: 43-49.

34. Kovalski G, Israel O, Keidar Z, Frenkel A, Sachs J, et al. (2007) Correction of heart motion due to respiration in clinical myocardial perfusion SPECT scans using respiratory gating. J Nucl Med 48: 630-636.

35. Sharir T, Ben-Haim S, Merzon K, Prochorov V, Dickman D, et al. (2008) High-speed myocardial perfusion imaging initial clinical comparison with conventional dual detector anger camera imaging. JACC Cardiovasc Imaging 1: 156-163.

36. Esteves FP, Raggi P, Folks RD, Keidar Z, Askew JW, et al. (2009) Novel solid-state-detector dedicated cardiac camera for fast myocardial perfusion imaging: multicenter comparison with standard dual detector cameras. $J$ Nucl Cardiol 16: 927-934.

37. Herzog BA, Buechel RR, Katz R, Brueckner M, Husmann L, et al. (2010) Nuclear myocardial perfusion imaging with a cadmium-zinc-telluride detector technique: optimized protocol for scan time reduction. J Nucl Med 51: 46-51.

38. Sharir T, Slomka PJ, Hayes SW, DiCarli MF, Ziffer JA, et al. (2010) Multicenter trial of high-speed versus conventional single-photon emission computed tomography imaging: quantitative results of myocardial perfusion and left ventricular function. J Am Coll Cardiol 55: 1965-1974.

39. Tsuchiya K, Takahashi I, Kawaguchi T, Yokoi K, Morimoto Y, et al. (2010) Basic performance and stability of a CdTe solid-state detector panel. Ann Nucl Med 24: 301-311.

40. Fukushima $\mathrm{Y}$, Kumita S, Kawaguchi T, Maruyama T, Kawasaki $\mathrm{Y}$, et al (2014) Nuclear myocardial perfusion imaging with a cadmium-telluride semiconductor detector gamma camera in patients with acute myocardial infarction. Ann Nucl Med 28: 646-655

41. Takahashi $Y$, Miyagawa M, Nishiyama Y, Ishimura H, Mochizuki T (2013) Performance of a semiconductor SPECT system: comparison with a conventional Anger-type SPECT instrument. Ann Nucl Med 27: 11-16.

42. Liu CJ, Cheng JS, Chen YC, Huang YH, Yen RF (2015) A performance comparison of novel cadmium-zinc-telluride camera and conventional SPECT/CT using anthropomorphic torso phantom and water bags to simulate soft tissue and breast attenuation. Ann Nucl Med 29: 342-350.

43. Herzog BA, Buechel RR, Husmann L, Pazhenkottil AP, Burger IA, et al. (2010) Validation of CT attenuation correction for high-speed myocardial perfusion imaging using a novel cadmium-zinc-telluride detector technique. J Nucl Med 51: 1539-1544

44. Caobelli F, Akin M, Thackeray JT, Brunkhorst T, Widder J, et al. (2015) Diagnostic accuracy of cadmium-zinc-telluride-based myocardial perfusion SPECT: impact of attenuation correction using a co-registered external computed tomography. Eur Heart J Cardiovasc Imaging. [In press].
45. Schroeder S, Achenbach S, Bengel F, Burgstahler C, Cademartiri F, et al. (2008) Cardiac computed tomography: indications, applications limitations, and training requirements: report of a Writing Group deployed by the Working Group Nuclear Cardiology and Cardiac CT of the European Society of Cardiology and the European Council of Nuclear Cardiology. Eur Heart J 29: 531-556.

46. Bartúnek J, Sys SU, Heyndrickx GR, Pijls NH, De Bruyne B (1995) Quantitative coronary angiography in predicting functional significance of stenoses in an unselected patient cohort. J Am Coll Cardiol 26: 328-334.

47. Schuijf JD, Wijns W, Jukema JW, Atsma DE, de Roos A, et al. (2006) Relationship between noninvasive coronary angiography with multi-slice computed tomography and myocardial perfusion imaging. J Am Coll Cardiol 48: 2508-2514.

48. Boden WE, O'Rourke RA, Teo KK, Hartigan PM, Maron DJ, et al. (2007) Optimal medical therapy with or without $\mathrm{PCl}$ for stable coronary disease. $\mathrm{N}$ Engl J Med 356: 1503-1516.

49. Group BDS, Frye RL, August P, Brooks MM, Hardison RM, Kelsey SF, et al. (2009) A randomized trial of therapies for type 2 diabetes and coronary artery disease. N Engl J Med 360: 2503-2515.

50. Klocke FJ, Baird MG, Lorell BH, Bateman TM, Messer JV, et al. (2003) ACC AHA/ASNC guidelines for the clinical use of cardiac radionuclide imaging-executive summary: a report of the American College of Cardiology/ American Heart Association Task Force on Practice Guidelines (ACC/AHA ASNC Committee to Revise the 1995 Guidelines for the Clinical Use of Cardiac Radionuclide Imaging). J Am Coll Cardiol 42: 1318-1333.

51. Iskander S, Iskandrian AE (1998) Risk assessment using single-photon emission computed tomographic technetium-99m sestamibi imaging. J Am Coll Cardiol 32: 57-62.

52. Ragosta M, Bishop AH, Lipson LC, Watson DD, Gimple LW, et al. (2007) Comparison between angiography and fractional flow reserve versus single-photon emission computed tomographic myocardial perfusion imaging for determining lesion significance in patients with multivessel coronary disease. Am J Cardiol 99: 896-902.

53. Yoshinaga K, Katoh C, Noriyasu K, Iwado Y, Furuyama H, et al. (2003) Reduction of coronary flow reserve in areas with and without ischemia on stress perfusion imaging in patients with coronary artery disease: a study using oxygen 15-labeled water PET. J Nucl Cardiol 10:275-283.

54. Lima RS, Watson DD, Goode AR, Siadaty MS, Ragosta M, et al. (2003) Incremental value of combined perfusion and function over perfusion alone by gated SPECT myocardial perfusion imaging for detection of severe three-vessel coronary artery disease. J Am Coll Cardiol 42: 64-70.

55. Gaemperli O, Schepis T, Koepfli P, Valenta I, Soyka J, et al. (2007) Accuracy of 64-slice CT angiography for the detection of functionally relevant coronary stenoses as assessed with myocardial perfusion SPECT. Eur J Nucl Med Mol Imaging 34: 1162-1171.

56. Falk E, Shah PK, Fuster V (1995) Coronary plaque disruption. Circulation 92: 657-671.

57. Dilsizian V, Bateman TM, Bergmann SR, Des Prez R, Magram MY, et al. (2005) Metabolic imaging with beta-methyl-p-[(123)!]-iodophenylpentadecanoic acid identifies ischemic memory after demand ischemia. Circulation 112: 2169-2174.

58. Kontos MC, Dilsizian V, Weiland F, DePuey G, Mahmarian JJ, et al. (2010) lodofiltic acid I 123 (BMIPP) fatty acid imaging improves initial diagnosis in emergency department patients with suspected acute coronary syndromes: a multicenter trial. J Am Coll Cardiol 56: 290-299.

59. Fukushima $\mathrm{Y}$, Toba M, Ishihara K, Mizumura S, Seino T, et al. (2008) Usefulness of 201TICI/123I-BMIPP dual-myocardial SPECT for patients with non-ST segment elevation myocardial infarction. Ann Nucl Med 22: 363-369.

60. Ishida Y, Yasumura Y, Nagaya N, Fukuchi K, Komamura K, et al. (1999) Myocardial imaging with 123I-BMIPP in patients with congestive heart failure. Int J Card Imaging 15: 71-77.

61. Budoff MJ, Dowe D, Jollis JG, Gitter M, Halamert E, et al. (2008) Diagnostic performance of 64-multidetector row coronary computed tomographic angiography for evaluation of coronary artery stenosis in individuals without known coronary artery disease: results from the prospective multicenter ACCURACY (Assessment by Coronary Computed Tomographic Angiography of Individuals Undergoing Invasive Coronary Angiography) trial. J Am Coll Cardiol 52: 1724-1732.
Int J Radiol Med Imag

ISSN: $2456-446 \mathrm{X}$
IJRMI, an open access journal

Volume 2. 2016. 113 
62. Hamon M, Morello R, Riddell JW, Hamon M (2007) Coronary arteries: diagnostic performance of 16- versus 64-section spiral CT compared with invasive coronary angiography--meta-analysis. Radiology 245: 720-731.

63. Sharma A, Arbab-Zadeh A (2012) Assessment of coronary heart disease by CT angiography: current and evolving applications. J Nucl Cardiol 19 : 796-806.

64. Morsbach F, Desbiolles L, Plass A, Leschka S, Schmidt B, et al. (2013) Stenosis quantification in coronary CT angiography: impact of an integrated circuit detector with iterative reconstruction. Invest Radiol 48: 32-40.

65. Motoyama S, Kondo T, Sarai M, Sugiura A, Harigaya H, et al. (2007) Multislice computed tomographic characteristics of coronary lesions in acute coronary syndromes. J Am Coll Cardiol 50: 319-326.

66. Motoyama S, Sarai M, Harigaya H, Anno H, Inoue K, et al. (2009) Computed tomographic angiography characteristics of atherosclerotic plaques subsequently resulting in acute coronary syndrome. J Am Coll Cardiol 54: 49-57.

67. Maurovich-Horvat $\mathrm{P}$, Hoffmann U, Vorpahl M, Nakano M, Virmani R, et al (2010) The napkin-ring sign: CT signature of high-risk coronary plaques? JACC Cardiovasc Imaging 3: 440-444.

68. Seifarth H, Schlett CL, Nakano M, Otsuka F, Károlyi M, et al. (2012) Histopathological correlates of the napkin-ring sign plaque in coronary $\mathrm{C} T$ angiography. Atherosclerosis 224: 90-96

69. Maurovich-Horvat $\mathrm{P}$, Schlett $\mathrm{CL}$, Alkadhi H, Nakano M, Otsuka F, et al (2012) The napkin-ring sign indicates advanced atherosclerotic lesions in coronary CT angiography. JACC Cardiovasc Imaging 5: 1243-1252.

70. Slomka PJ, Dey D, Duvall WL, Henzlova MJ, Berman DS, et al. (2012) Advances in nuclear cardiac instrumentation with a view towards reduced radiation exposure. Curr Cardiol Rep 14: 208-216.

71. Javadi MS, Lautamäki R, Merrill J, Voicu C, Epley W, et al. (2010) Definition of vascular territories on myocardial perfusion images by integration with true coronary anatomy: a hybrid PET/CT analysis. J Nucl Med 51: 198-203.

72. Nakajo H, Kumita S, Cho K, Kumazaki T (2005) Three-dimensiona registration of myocardial perfusion SPECT and CT coronary angiography Ann Nucl Med 19: 207-215.

73. Rispler S, Keidar Z, Ghersin E, Roguin A, Soil A, et al. (2007) Integrated single-photon emission computed tomography and computed tomography coronary angiography for the assessment of hemodynamically significant coronary artery lesions. J Am Coll Cardiol 49: 1059-1067.

74. Sato A, Nozato T, Hikita H, Miyazaki S, Takahashi Y, et al. (2010) Incremental value of combining 64-slice computed tomography angiography with stress nuclear myocardial perfusion imaging to improve noninvasive detection of coronary artery disease. J Nucl Cardiol 17: 19-26.

75. Gaemperli O, Schepis T, Valenta I, Husmann L, Scheffel H, et al. (2007) Cardiac image fusion from stand-alone SPECT and CT: clinical experience. J Nucl Med 48: 696-703.

76. Santana CA, Garcia EV, Faber TL, Sirineni GK, Esteves FP, et al. (2009) Diagnostic performance of fusion of myocardial perfusion imaging (MPI) and computed tomography coronary angiography. J Nucl Cardiol 16: 201 211

77. Slomka PJ, Cheng VY, Dey D, Woo J, Ramesh A, et al. (2009) Quantitative analysis of myocardial perfusion SPECT anatomically guided by coregistered 64-slice coronary CT angiography. J Nucl Med 50: 1621-1630.

78. van Werkhoven JM, Schuijf JD, Gaemperli O, Jukema JW, Boersma E, et al. (2009) Prognostic value of multislice computed tomography and gated single-photon emission computed tomography in patients with suspected coronary artery disease. J Am Coll Cardiol 53: 623-632.

79. Pazhenkottil AP, Nkoulou RN, Ghadri JR, Herzog BA, Buechel RR, et al (2011) Prognostic value of cardiac hybrid imaging integrating single-photon emission computed tomography with coronary computed tomography angiography. Eur Heart J 32: 1465-1471.

80. Pazhenkottil AP, Nkoulou RN, Ghadri JR, Herzog BA, Kuest SM, et al. (2011) Impact of cardiac hybrid single-photon emission computed tomography/ computed tomography imaging on choice of treatment strategy in coronary artery disease. Eur Heart J 32: 2824-2829.
81. Schaap J, de Groot JA, Nieman K, Meijboom WB, Boekholdt SM, et al. (2013) Hybrid myocardial perfusion SPECT/CT coronary angiography and invasive coronary angiography in patients with stable angina pectoris lead to similar treatment decisions. Heart 99: 188-194.

82. Hachamovitch R, Nutter B, Hlatky MA, Shaw LJ, Ridner ML, et al. (2012) Patient management after noninvasive cardiac imaging results from SPARC (Study of myocardial perfusion and coronary anatomy imaging roles in coronary artery disease). J Am Coll Cardiol 59: 462-474.

83. Budoff MJ, Shaw LJ, Liu ST, Weinstein SR, Mosler TP, et al. (2007) Longterm prognosis associated with coronary calcification: observations from a registry of 25,253 patients. J Am Coll Cardiol 49: 1860-1870.

84. Detrano R, Guerci AD, Carr JJ, Bild DE, Burke G, et al. (2008) Coronary calcium as a predictor of coronary events in four racial or ethnic groups. $\mathrm{N}$ Engl J Med 358: 1336-1345.

85. Madhavan MV, Tarigopula M, Mintz GS, Maehara A, Stone GW, et al. (2014) Coronary artery calcification: pathogenesis and prognostic implications. J Am Coll Cardiol 63: 1703-1714.

86. Rumberger JA, Simons DB, Fitzpatrick LA, Sheedy PF, Schwartz RS (1995) Coronary artery calcium area by electron-beam computed tomography and coronary atherosclerotic plaque area. A histopathologic correlative study. 92: 2157-2162

87. Greenland P, Bonow RO, Brundage BH, Budoff MJ, Eisenberg MJ, et al. (2007) ACCF/AHA 2007 clinical expert consensus document on coronary artery calcium scoring by computed tomography in global cardiovascular risk assessment and in evaluation of patients with chest pain: a report of the American College of Cardiology Foundation Clinical Expert Consensus Task Force (ACCF/AHA Writing Committee to Update the 2000 Expert Consensus Document on Electron Beam Computed Tomography) developed in collaboration with the Society of Atherosclerosis Imaging and Prevention and the Society of Cardiovascular Computed Tomography. J Am Coll Cardiol 49: 378-402.

88. Rozanski A, Gransar H, Wong ND, Shaw LJ, Miranda-Peats R, et al. (2007) Clinical outcomes after both coronary calcium scanning and exercise myocardial perfusion scintigraphy. J Am Coll Cardiol 49: 1352-1361.

89. Schepis T, Gaemperli O, Koepfli P, Namdar M, Valenta I, et al. (2007) Added value of coronary artery calcium score as an adjunct to gated SPECT for the evaluation of coronary artery disease in an intermediate-risk population. J Nucl Med 48: 1424-1430.

90. Ghadri JR, Pazhenkottil AP, Nkoulou RN, Goetti R, Buechel RR, et al. (2011) Very high coronary calcium score unmasks obstructive coronary artery disease in patients with normal SPECT MPI. Heart 97: 998-1003.

91. Einstein AJ, Moser KW, Thompson RC, Cerqueira MD, Henzlova MJ (2007) Radiation dose to patients from cardiac diagnostic imaging. Circulation 116: 1290-1305.

92. Husmann L, Valenta I, Gaemperli O, Adda O, Treyer V, et al. (2008) Feasibility of low-dose coronary CT angiography: first experience with prospective ECG-gating. Eur Heart J 29: 191-197.

93. Hausleiter J, Martinoff S, Hadamitzky M, Martuscelli E, Pschierer I, et al. (2010) Image quality and radiation exposure with a low tube voltage protocol for coronary CT angiography results of the PROTECTION II Trial. JACC Cardiovasc Imaging 3: 1113-1123.

94. Achenbach S, Marwan M, Ropers D, Schepis T, Pflederer T, et al. (2010) Coronary computed tomography angiography with a consistent dose below $1 \mathrm{mSv}$ using prospectively electrocardiogram-triggered high-pitch spiral acquisition. Eur Heart J 31: 340-346.

95. Buechel RR, Herzog BA, Husmann L, Burger IA, Pazhenkottil AP, et al. (2010) Ultrafast nuclear myocardial perfusion imaging on a new gamma camera with semiconductor detector technique: first clinical validation. Eur J Nucl Med Mol Imaging 37: 773-778.

96. Husmann L, Herzog BA, Gaemperli O, Tatsugami F, Burkhard N, et al. (2009) Diagnostic accuracy of computed tomography coronary angiography and evaluation of stress-only single-photon emission computed tomography/computed tomography hybrid imaging: comparison of prospective electrocardiogram-triggering vs. retrospective gating. Eur Heart J 30: 600-607.
Int J Radiol Med Imag

ISSN: $2456-446 \mathrm{X}$
IJRMI, an open access journal

Volume 2. 2016. 113 
Citation: Fukushima Y, Kumita S (2016) Cardiac SPECT/CT Imaging: CT Attenuation Correction and SPECT/CT Hybrid Imaging. Int J Radiol Med Imag 2: 113. doi: https://doi.org/10.15344/2456-446X/2016/113

Page 12 of 12

97. Palyo R, Sinusas A, Liu YH (2016) High-Sensitivity and High-Resolution SPECT/CT Systems Provide Substantial Dose Reduction without Compromising Quantitative Precision for Assessment of Myocardial Perfusion or Function. J Nucl Med [In press].

98. Diamond GA, Forrester JS (1979) Analysis of probability as an aid in the clinical diagnosis of coronary-artery disease. N Engl J Med 300: 1350-1358.

99. Tonino PA, Fearon WF, De Bruyne B, Oldroyd KG, Leesar MA, et al. (2010) Angiographic versus functional severity of coronary artery stenoses in the FAME study fractional flow reserve versus angiography in multivessel evaluation. J Am Coll Cardiol 55: 2816-2821.

100. Stahli BE, Bonassin F, Goetti R, Kuest SM, Frank M, et al. (2012) Coronary computed tomography angiography indicates complexity of percutaneous coronary interventions. J Invasive Cardiol 24: 196-201.

101. FitzGibbon GM, Leach AJ, Kafka HP, Keon WJ (1991) Coronary bypass graft fate: long-term angiographic study. J Am Coll Cardiol 17: 1075-1080.

102. van Brussel BL, Plokker HW, Voors AA, Ernst SM, Kelder HC (1997) Progression of atherosclerosis after venous coronary artery bypass graft surgery: a 15-year follow-up study. Cathet Cardiovasc Diagn 41: 141-150.

103. Mathew V, Clavell AL, Lennon RJ, Grill DE, Holmes DR, Jr. (2000) Percutaneous coronary interventions in patients with prior coronary artery bypass surgery: changes in patient characteristics and outcome during two decades. Am J Med 108: 127-135.

104. Abe H, Iguchi N, Utanohara $\mathrm{Y}$, Inoue $\mathrm{K}$, Takamisawa I, et al. (2014) Noninvasive diagnosis of coronary artery disease by 123I-BMIPP/201TICl dual myocardial SPECT in patients with heart failure. Int J Cardiol 176: 969-974.

105. Miyachi H, Kumita S, Tanaka K (2013) PET/CT and SPECT/CT cardiac fusion imaging in a patient with takotsubo cardiomyopathy. Eur Heart J 34: 397. 